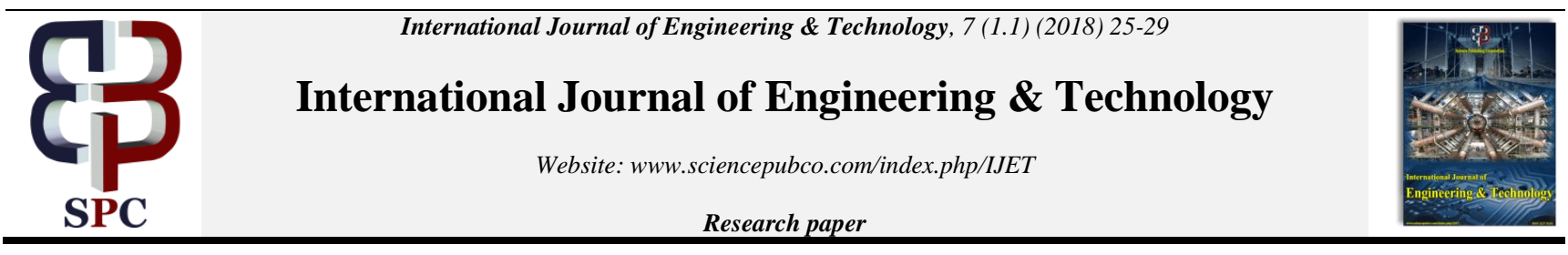

\title{
Implementation of hybrid filter technique for noise removal from medical images
}

\author{
Shruti Bhargava Choubey*, S.P.V. Subba Rao \\ Sreenidhi Institute of Science \& Technology, Hyderabad. \\ *Corresponding author E-mail: shrutibhargava@sreenidhi.edu.in
}

\begin{abstract}
Image denoising is used to eliminate the noise while retaining as much as possible the important signal features. The function of image denoising is to calculate approximately the original image form the noisy data. Image denoising still remains the challenge for researchers because noise removal introduces artifacts and causes blurring of the images. Image denoising has become an essential exercise in medical imaging especially the Magnetic Resonance Imaging (MRI). MR images are typically corrupted with noise, which hinder the medical diagnosis based on these images. The presence of noise not only causes as undesirable visual quality as well as lowers the visibility of low contrast objects. in this paper noise removal approach has proposed using hybridization of three filter with DWT method.
\end{abstract}

Results calculated in terms of PSNR,MSE \& TIME

Keywords: PSNR,MSE,DWT,BVS,MAPE,MD.

\section{Introduction}

In day-to-day life, digital images have a key role in the area of computer aided tomography, aerial communications, telecommunication images, synthetic aperture radar, geographical information systems, astronomy etc. In diverse fields, mentioned above, scientists are facing the problem of recovering original images from incomplete, indirect and noisy images. Images get corrupted during acquisition by camera sensors, receivers, environmental conditions, improper lightning, undesirable view angle etc., [2]. A noisy image appears as spotted, granular, hoary image. Therefore, the problem of recovering an original image from noisy image has received an ever increasing attention in recent years [3]. The recovery of image can be accomplished by image denoising, a process of estimating the desired image from a corrupted image [4,5]. Image denoising is a course of action in digital image processing aimed at the removal of noise. The most important reason to diminish noise is that extraneous features will otherwise cause successive errors in recognition. Another motivation is that noise reduction reduces the size of the image file, and this in contrast reduces the time required for successive processing and storage. The purpose in the design of a filter to diminish noise is that it remove as much of the noise as possible while maintaining all of the image qualities. Noise is inevitably introduced to medical images due to various factors in medical imaging which degrades the quality of images, not clearly visible boundaries and structural details are not clearly visible, thus causes difficulties to medical diagnosis. Also there is a tradeoff between time/spatial resolution and signal to noise ratio (SNR). Practically acquisition time in medical imaging is limited due to patient comfort and system requirement. Therefore, fast imaging is needed. But when the time resolution is improved, the noise may cause the quality of images to be degraded, blurring boundaries and suppressing structural details, thus bring difficulties to medical diagnosis. Therefore, the important factor that medical image de-noising adhere is to remove the noise while preserving important features [8].

\section{Literature survey}

THE literature review summarizes, interprets and evaluates existing "literature" (published or available material) in order to establish current knowledge of a subject. The reason for doing literature survey is to relate to ongoing research to develop that knowledge. SOME paper investigates the suitability of different wavelet bases and the size of different neighbourhood on the performance of image de-noising algorithms in terms of PSNR and the image de-noising using discrete wavelet transform is analyzed. The experiments were conducted to study the suitability of different wavelet bases and also different window sizes. Among all discrete wavelet bases, coif let performs well in image denoising[14].

D.Giaouris, J.W.Finch [15] showed that the denoising scheme based on the WT did not distort the signal and the noise component after the process was found to be small. But this process imposed a certain delay on the signal and was relatively complicated. In fixed frequency case, no improvement had been noted.

Manish Goyal, Glenetan singh sekhon [3] In this paper ,a hybrid method is proposed for removing speckle noise from the image. Proposed method consist of two wavelet thresholding techniques: first technique by using statistical method and second technique based on bayes threshold. Result of both method is averaged and apply threshold for soft thresholding .for post processing wiener filter is used. It has been observed that combination of this method does perform better than the existing techniques. In wavelet based techniques edge preservation is also good and better speckle noise 
suppression.

Sudipta Roy, Nidul Sinha, Asoke K. Sen [6] In this paper A new model based on the hybridization of wavelet and bilateral filters for denoising of variety of noisy images is presented. The model is experimented on standard images, like $\mathrm{x}$-ray images, ultrasound and astronomical telescopic images and the performances are evaluated in terms of peak signal to noise ratio (PSNR) and image quality index (IQI). Results demonstrate that use of bilateral filters in combination with wavelet thresholding filters on subbands of a decomposed image deteriorates the performance.

\section{Proposed method}

Given a noisy signal $\mathrm{y}=\mathrm{x}+\mathrm{n}$ where $\mathrm{x}$ is the desired signal and $\mathrm{n}$ is independent and identically distributed (i.i.d) Gaussian noise $N$ $\left(0, \sigma^{2}\right), \mathrm{y}$ is first decomposed into a set of wavelet coefficients $\mathrm{w}=$ $W[y]$ consisting of the required coefficient $\theta$ and noise coefficient $\mathrm{n}$. By applying a suitable threshold value $\mathrm{T}$ to the wavelet coefficients, the desired coefficient $\theta=\mathrm{T}[\mathrm{w}]$ can be obtained; Lastly an inverse transform on the desired coefficient $\theta$ will generate the denoise signal $\mathrm{x}=W^{\mathrm{T}}[\theta]$.

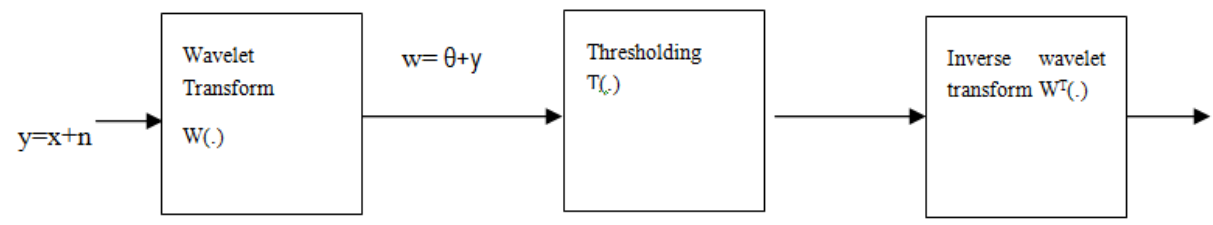

Fig. 1: Block diagram for DWT based denoising framework

A similar approach can be applied to a speckle SAR image. The wavelet decomposition process is iterated with following approximations being decayed in turn, so that the image is broken down and represented by a small number coarser component in the lower spectral band (LL block) and a large number of detailed components in the higher spectral band (LH, HL and HH blocks). In broad-spectrum a signal has its energy concentrated in a small numeral of coefficients, while noise has its energy spread across over a large number of coefficients. Hence, through suitable thresholding or wavelet shrinkage of the higher spectral bands components (where the noise predominantly lies) we can greatly reduce or remove the noise speckle of the image in a wavelet domain. Since the noise characteristics can be different in each higher spectral block, each block will have to be thresholded separately according to its local noise variance. Finally, the thresholded coefficients are used in a wavelet reconstruction process to retrieve the speckle-reduced image with little loss of detail.

\section{Algorithm for Denoising}

I=Input Image

Taking I into the Denoising Block

Convert I from RGB to Gray Colour MAP say Igray

(Because the multidimensional matrix not supported by many digital filters \& functions)

Let, $d w t=$ Wavelet transform and THfilt=Wavelet filtering with respect to threshold

$(c A, c H, c V, c D)=d w t$ (Igray);

Here, $\mathrm{cA}, \mathrm{cH}, \mathrm{cV}$ and $\mathrm{cD}$ are approximation, Horizontal, Vertical and Diagonal Coefficients respectively.

Update the approximation coefficients by filtering it.

$c A=T H f i l t(c A)$;

THfilt is the thresholding function that we considered, by taking following points as a filtering criteria

- Eliminate in the wavelet representation those elements with small coefficients, and

- Decrease the impact of elements with large coefficients.

- In mathematical terms, all we are doing is thresholding the absolute value of wavelet coefficients by an appropriate function.

Similar operation will be perform for multi-level decomposition For second level

$(c A l, c H, c V, c D)=d w t(c A)$;

$c A l=\operatorname{THfilt}(c A 1)$

For third level $(c A 2, c H, c V, c D)=d w t(c A 1)$;

$c A 2=$ THfilt $(c A 2)$;

After the transformation procedure, we have to apply inverse transform on the input medical image Let, idwt=Inverse Wavelet Transform of data
$Y 2=i d w t$ (with respect to $c A 2 \&$ other parameters are kept same), $Y 1=i d w t$ (with respect to $c A 1 \&$ other parameters are kept same); $Y=i d w t$ (with respect to $c A$ and $c D u \&$ other parameters are kept same);

Here, Updated Diagonal coefficients are calculated as $c D u=$ mean of $c D \& c H$

Let, median, average and diffusion are median, average and diffusion filters for data filtering.

$Y m=\operatorname{Median}(Y)$;

A median filter is more effective than convolution when the goal is to simultaneously reduce noise and preserve edges.

Create a filter structure for average filter, Say $F S a$, after that, we have to perform multidimensional filtering on median filtered data, according to the specified options for average filter.

$Y a=$ Filter $(Y m, F S a)$;

Numerical gradient calculations is perform from average filtered output, say by function Gradient

$f x, f y=$ Gradient $(Y a)$

After that, we have to calculate discrete Laplacian of the average filtered image, in order to pass it through diffusion approximation with two dimensional gradient functions. Say, Diffusion is the function involving whole process of anisotropic diffusion which we have to apply on average filtered data, reducing image noise without removing significant parts of the image content, typically edges, lines or other details that are important for the interpretation of the image.

$Y d=\operatorname{Diffusion}(Y a)$;

Here, Yd is the final output that we are taking from denoising block which we use further in segmentation block As a consequence, the resulting medical images preserve linear structures while at the same time smoothing is made along these structures. Both these cases can be described by a generalization of the usual diffusion equation where the diffusion coefficient, instead of being a constant scalar, is a function of image position and assumes a matrix (or tensor).

\section{Result \& discussion}

As a first we implemented basic discrete wavelet transform based denoising and calculated the efficiency in terms of MSE and PSNR. In order to overcome the ill influence of noise and shading, there is a need to take them into consideration when selecting the threshold being used. On the other hand, this is an impossible mission in a global context, since no one threshold can fit the entire image. This leads to the conclusion, that a more local threshold must be used. The locality property can allow a few cautious assumptions, and according to them produce a suitable threshold for the pixels in the environment. 


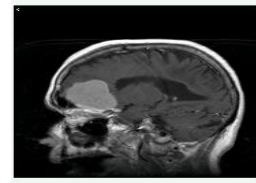

Original Medical Image
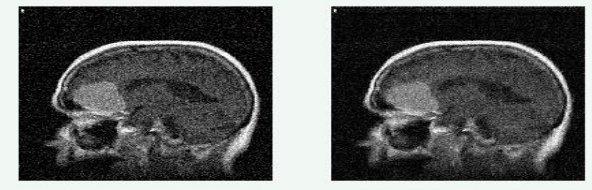

Hoised Medical Image

Denoised Medical Image
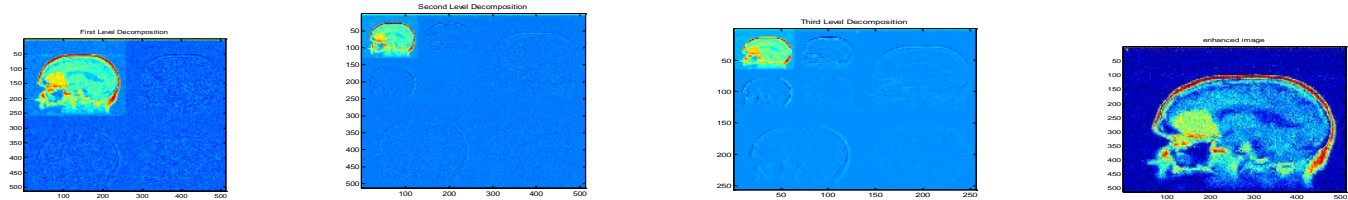

Fig. 2: (a) (b) (c) Different level of wavelet decomposition. (d) enhanced image

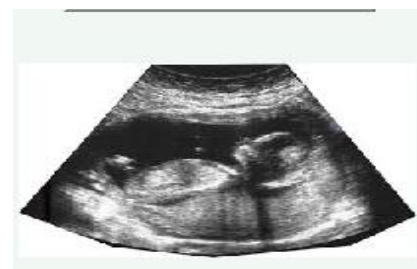

Original Medical Image

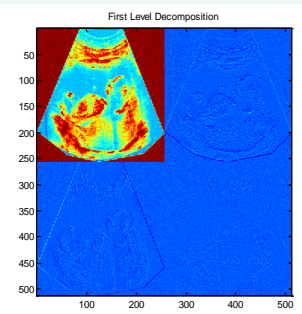

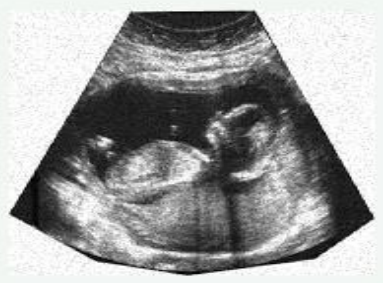

Hoised Medical Image

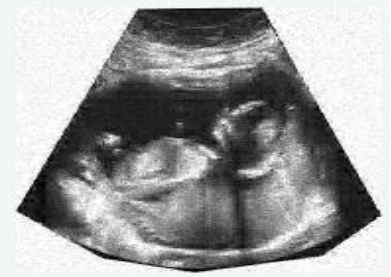

Denoised Medical Image

Fig. 3: (a) (b) (c) Different level of wavelet decomposition (d) Enhanced image
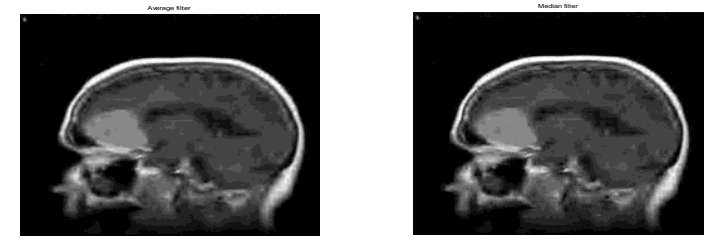

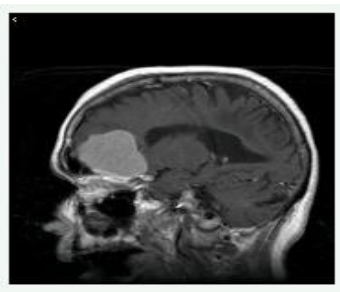

Original Medical Image

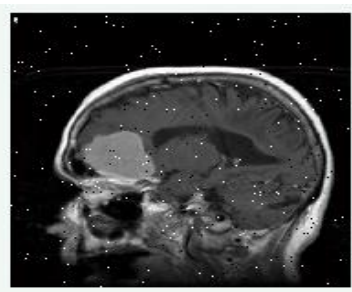

Hoised Medical Image
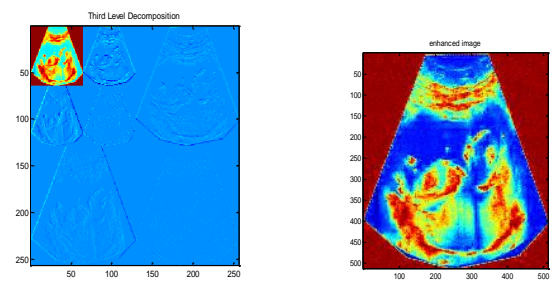

Fig. 4: Resultant image after a (Median filter), b (Average filter), c (Diffusion filter)final denoised image)
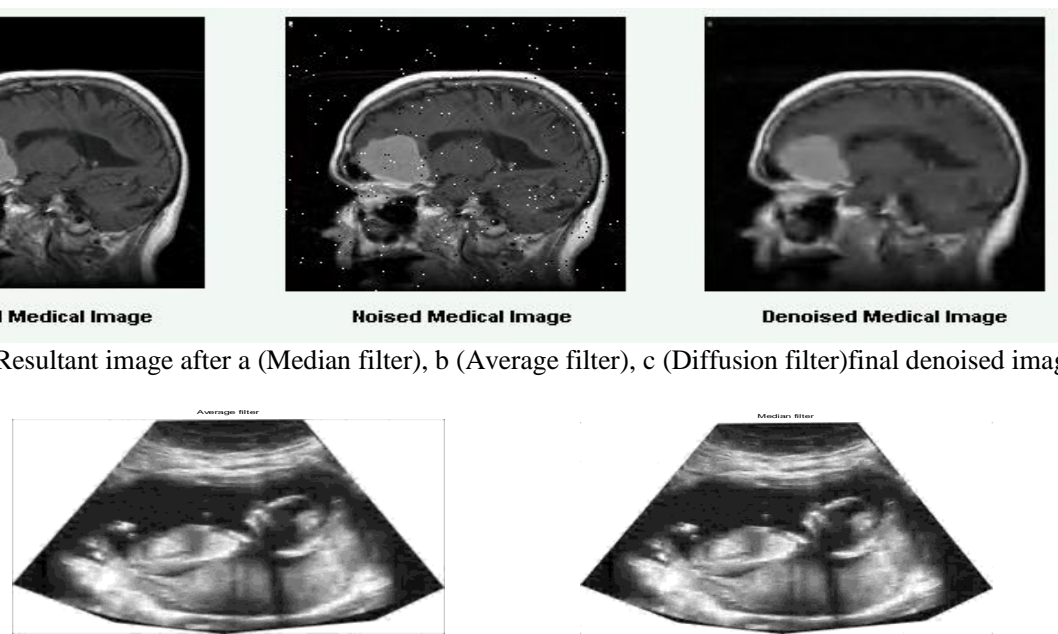

Denoised Medical Image

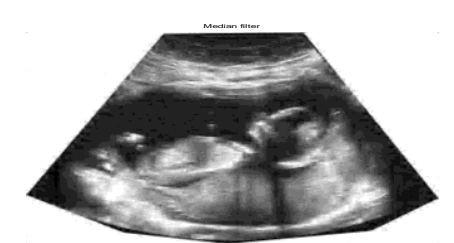

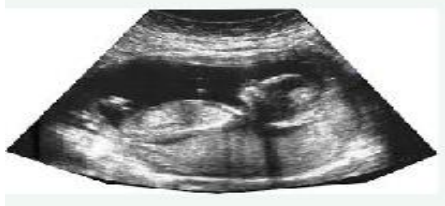

Original Medical Image

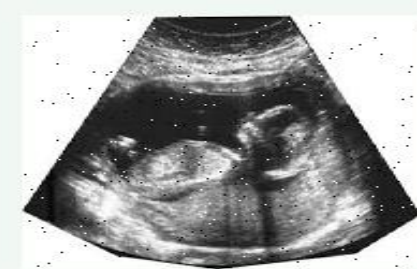

Hoised Medical Image

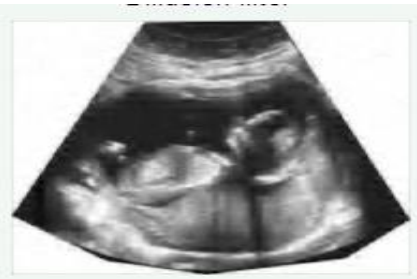

Denoised Medical Image 
Fig. 5: Resultant image after a (Median filter), b (Average filter), c (Diffusion filter final denoised image)

Table 1: Table Showing Different PSNR, MSE, WPSNR, SSIM and Run Time for Image

\begin{tabular}{|l|l|l|l|l|l|}
\hline Salt \& Pepper & PSNR & MSE & WPSNR & SSIM & TIME \\
\hline DWT & 33.98 & 25.96 & 34.93 & 0.6171 & 9.33 \\
\hline Filters & 34.25 & 24.41 & 32.85 & 0.779 & 4.15 \\
\hline Gaussion & PSNR & MSE & WPSNR & SSIM & TIME \\
\hline DWT & 31.57 & 45.29 & 32.65 & 0.5038 & 0.577 \\
\hline Filters & 34.26 & 24.36 & 32.9 & 0.5684 & 1.147 \\
\hline Speckle & PSNR & MSE & WPSNR & SSIM & TIME \\
\hline DWT & 33.44 & 29.43 & 34.83 & 0.8096 & 0.5212 \\
\hline Filters & 34 & 25.86 & 32.56 & 0.8292 & 1.0888 \\
\hline Poisson & PSNR & MSE & WPSNR & SSIM & TIME \\
\hline DWT & 33.32 & 30.23 & 35.03 & 0.8094 & 0.5388 \\
\hline Filters & 34.05 & 25.56 & 32.67 & 0.8285 & 1.066 \\
\hline
\end{tabular}

Table 2: Table Showing Different PSNR, MSE, WPSNR, SSIM and Run Time for Image 2

\begin{tabular}{|l|l|l|l|l|l|}
\hline Salt \& pepper & PSNR & MSE & WPSNR & SSIM & TIME \\
\hline DWT & 31.83 & 42.59 & 31.75 & 0.6969 & 1.76 \\
\hline Filters & 31.71 & 43.81 & 29.96 & 0.8112 & 1.304 \\
\hline Bivariate Shrinkage & 47.059 & 1.274 & 38.12 & 0.8302 & 0.2085 \\
\hline Dual Tree with CWT & 47.03 & 1.288 & 38.16 & 0.831 & 0.6632 \\
\hline Gaussion & PSNR & MSE & WPSNR & SSIM & TIME \\
\hline DWT & 28.15 & 91.43 & 29.26 & 0.766 & 0.5541 \\
\hline Filters & 29.81 & 67.85 & 28.69 & 0.8039 & 1.0996 \\
\hline Bivariate Shrinkage & 30.12 & 63.14 & 33.29 & 0.8974 & 0.2229 \\
\hline Dual Tree with CWT & 30.15 & 59.97 & 33.56 & 0.9169 & 0.67 \\
\hline Speckle & PSNR & MSE & WPSNR & SSIM & TIME \\
\hline DWT & 29.16 & 78.81 & 30.15 & 0.7983 & 0.9164 \\
\hline Filters & 29.42 & 74.18 & 28.46 & 0.8091 & 1.153 \\
\hline Bivariate Shrinkage & 30.12 & 56.33 & 33.59 & 0.9336 & 0.2878 \\
\hline Dual Tree with CWT & 30.7 & 55.23 & 33.61 & 0.9385 & 5.4801 \\
\hline Poisson & PSNR & MSE & WPSNR & SSIM & TIME \\
\hline DWT & 30.12 & 63.2 & 31.6 & 0.7988 & 0.5402 \\
\hline Filters & 30.81 & 53.84 & 29.3 & 0.8106 & 1.092 \\
\hline Bivariate Shrinkage & 33.85 & 26.79 & 37.81 & 0.9705 & 0.2231 \\
\hline Dual Tree with CWT & 34.17 & 24.89 & 38 & 0.977 & 0.661 \\
\hline
\end{tabular}

Comparison with existing method

Table 3: Table Showing Different PSNR, MSE, WPSNR, SSIM

\begin{tabular}{|l|l|l|l|l|}
\hline Method/parameter & PSNR & MSE & SSIM & ET \\
\hline HF (using CWM) & 25.49234 & 34.84196 & 0.98692 & 2.434407 \\
\hline Proposed method & 33.98 & 25.96 & .9979 & 1.7435 \\
\hline
\end{tabular}

\section{Conclusion}

However selection of the actual denoising procedure plays an important role, it is essential develop to experiment and compare the methods. Finally it is also possible to combine our method with other to get high quality of result. Along with these points we combine various filters namely, average filter, median filter and diffusion filter with DWT. The result of wavelet threshold are fed into the sequence of filter (average, median and diffusion) and the image denoising result are found to be improved on most of the image type. As the preprocessing filter is designed in the wavelet domain, it provides a composite effect on improving the denoising performance of given hybrid method at high frequencies. Experimental results prove that the method not only improves the denoising performance in terms of PSNR, SSIM, and visual presentation, but also it reduces the execution time required for denoising.

\section{References}

[1] Balasubramanian G, Chilambuchelvan A, Vijayan S \& Gowrison G, "Performance Improvement of Average Based Spatial Filters through Multilevel Preprocessing using Wavelets", IEEE Signal Processing Letters, Vol.22, No.10, (2015).
[2] Ruikar SD \& Doye DD, "Wavelet Based Image Denoising Technique" International Journal of Advanced Computer Science and Applications, Vol.2, No.3, (2011).

[3] Raj A, Srivastava A \& Bhateja V, "Computer aided detection of brain tumor in magnetic resonance images", International Journal of Engineering and Technology, Vol.3, No.5, (2011).

[4] Goyal M \& sekhon GS, "Hybrid Threshold Technique for Speckle Noise Reduction using wavelets for Grey scale images", IJCST Vol.2, No.2, (2011)

[5] Mohideen K, Perumal A, Krishnan N \& Sathik M, "Image Denoising And Enhancement Using Multiwavelet With Hard Threshold In Digital Mammographic Images", International Arab Journal of e-Technology, Vol.2, No.1, (2011).

[6] Vidhyalavanya R \& Madheswaran M, "An Improved Denoising Algorithm using Parametric Multiwavelets for Image Enhancement", International Journal of Advanced Science and Technology, Vol.16, (2010).

[7] Roy S, Sinha N \& Sen AK, "A new hybrid image denoising methode", International Journal of Information Technology and Knowledge Management, Vol.2, No.2, pp.491-497, (2010).

[8] Patil SS, Patil AB, Deshmukh SC \& Chavan MN, "Wavelet Shrinkage Techniques for Images", International Journal of Computer Applications, Vol.7, No.1, (2010)

[9] Ruikar S \& Doye DD, "Image Denoising Using Wavelet Transform", International Conference on Mechanical and Electrical Technology, (2010).

[10] Bhat JS, Jagadale BN \& Lakshminarayan HK, "Image De-noising with an Optimal Threshold using Wavelets", International Conference on Image and signal processing, (2010). 
[11] Liu Wei, "New Method for Image Denoising while Keeping Edge Information", $2^{\text {nd }}$ International conference on image processing CISP, (2009).

[12] Lakshmi, K., Surendar, A. "Verification of axiprotocol using system Verilog", (2017), International Journal of Mechanical Engineering and Technology, 8 (5), pp. 588-595.

[13] Surendar, A., Kavitha, M. "Secure patient data transmission in sensor networks", (2017), Journal of Pharmaceutical Sciences and Research, 9 (2), pp. 230-232.

[14] Surendar, A.'FPGA based parallel computation techniques for bioinformatics applications",(2017) International Journal of Research in Pharmaceutical Sciences, 8 (2), pp. 124-128.

[15] Surendar, A.'Evolution of gait biometric system and algorithms- A review" (2017) Biomedical and Pharmacology Journal, 10 (1), pp. 467-472.

[16] Giaouris D \& Finch JW, "Denoising using wavelets on electric drive applications", School of Electrical, Electronic \& Computer Engineering, Newcastle University, Newcastle upon Tyne NE1 $7 R U$, (2007).

[17] Balster EJ, Zheng YF \& Ewing RL, "Feature-Based Wavelet Shrinkage Algorithm for Image Denoising”, IEEE Transactions On Image Processing, Vol.14, No.12, (2005).

[18] Starck JL, Fadili J \& Murtagh F, "The undecimated wavelet decomposition and its reconstruction", IEEE Transactions on Image Processing, Vol.16, No.2, pp.297-309, (2007).

[19] Shui PL \& Zhao YB, "Image denoising algorithm using doubly local Wiener filtering with block-adaptive windows in wavelet domain", Signal Processing, Vol.87, No.7, pp.1721-1734, (2007).

[20] Achim A \& Kuruo glu EE, "Image Denoising Using Bivariate $\alpha$ Stable Distributions in the Complex Wavelet Domain", IEEE Signal Processing Letters, Vol.12, No.1, (2005). 